\title{
Wave-induced fluid flow in random porous media: Attenuation and dispersion of elastic waves
}

\author{
Tobias M. Müller ${ }^{\mathrm{a})}$ and Boris Gurevich ${ }^{\mathrm{b})}$ \\ Department of Exploration Geophysics, Curtin University, ${ }^{\mathrm{c})}$ Perth, WA 6845 Australia
}

(Received 10 June 2004; revised 26 November 2004; accepted 1 March 2005)

\begin{abstract}
A detailed analysis of the relationship between elastic waves in inhomogeneous, porous media and the effect of wave-induced fluid flow is presented. Based on the results of the poroelastic first-order statistical smoothing approximation applied to Biot's equations of poroelasticity, a model for elastic wave attenuation and dispersion due to wave-induced fluid flow in 3-D randomly inhomogeneous poroelastic media is developed. Attenuation and dispersion depend on linear combinations of the spatial correlations of the fluctuating poroelastic parameters. The observed frequency dependence is typical for a relaxation phenomenon. Further, the analytic properties of attenuation and dispersion are analyzed. It is shown that the low-frequency asymptote of the attenuation coefficient of a plane compressional wave is proportional to the square of frequency. At high frequencies the attenuation coefficient becomes proportional to the square root of frequency. A comparison with the 1-D theory shows that attenuation is of the same order but slightly larger in 3-D random media. Several modeling choices of the approach including the effect of cross correlations between fluid and solid phase properties are demonstrated. The potential application of the results to real porous materials is discussed. (c) 2005 Acoustical Society of America. [DOI: 10.1121/1.1894792]
\end{abstract}

PACS numbers: 43.20.Jr, 43.20.Gp, 43.20.-f, 43.20.Px, 43.20.Wd [JJM] Pages: 2732-2741

\section{INTRODUCTION}

One major cause of elastic wave attenuation in heterogeneous porous media is wave-induced flow of the pore fluid between heterogeneities of various scales. It is believed that for frequencies below $1 \mathrm{kHz}$ most important is the waveinduced flow between mesoscopic inhomogeneities, which are large compared with the typical individual pore size, but small compared to the wavelength. Various laboratory experiments in some natural porous materials provide evidence for the presence of centimeter-scale (mesoscopic) heterogeneities. ${ }^{1,2}$ Attenuation and dispersion due to mesoscopic flow can be modeled using Biot's equations of poroelasticity with spatially varying coefficients. ${ }^{3}$

The simplest model of mesoscopic heterogeneities is a horizontally layered (1-D) structure. In such structures an elastic wave passing perpendicular to layers causes an "inter-layer" flow, that is the flow of the pore fluid from more compressible into stiffer layers during a compression cycle of the wave (and vice versa during extension). Elastic wave attenuation and dispersion due to interlayer flow were first studied for structures with periodic stratification. ${ }^{4,5}$ More recently, Gurevich and Lopatnikov ${ }^{6}$ and Gelinsky et al. ${ }^{7}$ analyzed attenuation and dispersion for structures with random layering. These 1-D studies revealed that frequency dependencies of attenuation and velocity in periodic and random layered structures were somewhat different. In both situations attenuation and dispersion have their maximum at a frequency $\omega_{\max }$ where (typical) layer thickness equals the

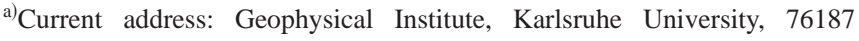
Karlsruhe, Germany, Electronic address: Tobias.Mueller@gpi.unikarlsruhe.de

${ }^{b}$ Electronic address: boris.gurevich@geophy.curtin.edu.au

${ }^{c}$ URL: http://www.geophysics.curtin.edu.au
}

fluid diffusion length (that is, the wavelength of Biot's slow wave). Furthermore, at higher frequencies, $\omega \gg \omega_{\max }$ attenuation (expressed through the reciprocal quality factor $Q^{-1}$ ) for both periodic and random layers has the same asymptotic behavior $Q^{-1} \propto \omega^{-1 / 2}$. However, attenuation is different in the low-frequency limit $\omega \ll \omega_{\max }$ : it is proportional to frequency $\omega$ for periodic layering and to $\sqrt{\omega}$ for random layering. These findings underline the importance of spatial distribution of inhomogeneities for modeling mesoscopic-flow attenuation and dispersion.

The situation is naturally more complex in porous materials with three-dimensional inhomogeneities. In such media the behavior of attenuation as a function of frequency depends on the distribution and shape of inhomogeneities. However, recently Johnson, ${ }^{8}$ Pride and Berryman, ${ }^{9}$ and Pride et al. ${ }^{10}$ showed that in porous media with a regular distribution of identical inhomogeneities of any fixed shape, the reciprocal quality factor $Q^{-1}$ scales with $\omega$ at low frequencies and with $\omega^{-1 / 2}$ for high frequencies. In real porous composites heterogeneities are more likely to have a random spatial distribution. Given the 1-D results quoted earlier, it is therefore natural to ask how the random distribution of inhomogeneities will influence the magnitude and frequency dependency of wave attenuation. To address this question, Müller and Gurevich ${ }^{11}$ developed a theory for elastic wave propagation in a fluid-saturated porous medium with random distribution of 3-D inhomogeneities. Applying the method of statistical smoothing ${ }^{12}$ to Biot's equations of poroelasticity with spatially variable coefficients, they derived an explicit expression for a complex-valued, effective wave number of a compressional wave ( $P$-wave) which accounts for the effect of wave-induced flow.

In this paper we use the results of Müller and Gurevich ${ }^{11}$ (referred below as paper I) to analyze the magnitude and 
frequency dependence of attenuation and velocity dispersion caused by wave-induced fluid flow in poroelastic random media. Specifically, we derive closed-form expressions for elastic wave attenuation and dispersion as functions of the correlation properties of the inhomogeneities (Sec. II). We show that these wave field attributes are mutually related by the Kramers-Kronig relations, and therefore represent a causal pair (Sec. III). For most commonly used correlation models we obtain simple, analytic expressions for attenuation and dispersion and demonstrate their behavior using numerical examples (Sec. IV). We also analyze the low- and high-frequency asymptotic behavior of both velocity and attenuation (Sec. V). Finally, in Sec. VI we discuss our results in the light of existing theories for wave propagation in poroelastic media, in thermoelastic media, and in suspensions of solid particles in a viscous fluid. The conclusions are presented in Sec. VII.

\section{ATTENUATION AND DISPERSION DUE TO WAVE- INDUCED FLOW}

According to paper I, the effective $P$-wave number $\bar{k}_{p}$ in 3 -D poroelastic random media can be written as a sum of the homogeneous background $P$-wave number $k_{p}$ and a correction term which accounts for the conversion scattering from fast into slow $P$-waves due to the presence of randomly distributed inhomogeneities

$$
\bar{k}_{p}=k_{p}\left(1+\Delta_{2}+\Delta_{1} k_{p s}^{2} \int_{0}^{\infty} r B(r) \exp \left[\mathrm{i} k_{p s} r\right] d r\right),
$$

where $\Delta_{1}$ and $\Delta_{2}$ are the dimensionless coefficients

$$
\begin{aligned}
\Delta_{1}= & \frac{\alpha^{2} M}{2 P_{d}}\left(\sigma_{H H}^{2}-2 \sigma_{H C}^{2}+\sigma_{C C}^{2}+\frac{32}{15} \frac{G^{2}}{H^{2}} \sigma_{G G}^{2}\right. \\
& \left.-\frac{8}{3} \frac{G}{H} \sigma_{H G}^{2}+\frac{8}{3} \frac{G}{H} \sigma_{G C}^{2}\right), \\
\Delta_{2}= & \Delta_{1}+\frac{1}{2} \sigma_{H H}^{2}-\frac{4}{3} \frac{G}{H} \sigma_{H G}^{2}+\left(\frac{4 G}{H}+1\right) \frac{4}{15} \frac{G}{H} \sigma_{G G}^{2},
\end{aligned}
$$

and $k_{p s}$ denotes the wave number of Biot's slow wave

$$
k_{p s}=\sqrt{\frac{i \omega \eta}{\kappa_{0} N}} .
$$

In Eqs. (2) and (3) $P_{d}$ and $H$ are respectively dry and saturated $P$-wave moduli of the background material, which are related by the Gassmann equation

$$
H=P_{d}+\alpha^{2} M,
$$

where

$$
M=\left[(\alpha-\phi) / K_{g}+\phi / K_{f}\right]^{-1},
$$

$\alpha=1-K_{d} / K_{g}$ is the Biot-Willis coefficient, $\phi$ is background porosity, and $N=M P_{d} / H$ while $K_{g}, K_{d}$, and $K_{f}$ denote the bulk moduli of the solid phase, the drained frame, and the pore fluid, respectively. The shear modulus is denoted as $G$. In Eq. (4) $\omega$ is angular frequency, $\kappa_{0}$ is the background permeability, and $\eta$ is the viscosity of the pore fluid. Equation
(1) was obtained by assuming that the parameters $H, G$, and $C=\alpha M$ have a random component. The correlation properties of the random inhomogeneities are characterized by the normalized correlation function $B(r)$, which for the three random functions $H, G$, and $C$ assumes the same functional form. The variances of the relative fluctuations are denoted as $\sigma_{H H}^{2}, \sigma_{G G}^{2}$, and $\sigma_{C C}^{2}$. The cross variance of the relative fluctuations are $\sigma_{H G}^{2}, \sigma_{H C}^{2}$, and $\sigma_{G C}^{2}$. Approximation (1) for the effective $P$-wave number has a restricted range of applicability, which can be expressed through the conditions (for a detailed analysis see paper I)

$$
\max \left\{\Delta_{1}\left(\left|k_{p s}\right| a\right)^{2}, \Delta_{2}\right\} \ll 1
$$

and

$$
a^{2} \gg \frac{\kappa_{0} N}{\eta \omega_{B}},
$$

where $\omega_{B}=\phi \eta /\left(\kappa_{0} \rho_{f}\right)$ is the characteristic Biot frequency with $\rho_{f}$ denoting fluid density and $a$ the correlation length, that is, a characteristic length scale of the inhomogeneities. Condition (7) expresses the weak-contrast assumption which is necessary for the derivation of the effective $P$-wave number, whereas condition (8) arises from the use of the lowfrequency approximation to Biot's equations of poroelasticity.

Equation (1) for the effective wave number enables us to derive expressions for the attenuation and dispersion due to the presence of mesoscopic inhomogeneities. By definition, the real part of $\bar{k}_{p}$ yields the phase velocity

$$
\begin{aligned}
v(\omega)= & \omega / \Re\left\{\bar{k}_{p}\right\}=v_{0}\left[1-\Delta_{2}+2 \Delta_{1} \bar{k}^{2}\right. \\
& \left.\times \int_{0}^{\infty} r B(r) \exp [-\bar{k} r] \sin (\bar{k} r) d r\right],
\end{aligned}
$$

where $v_{0}$ is the constant background $P$-wave velocity defined as $v_{0}=\sqrt{H / \rho}$ ( $\rho$ is the bulk density) and $\bar{k}$ denotes the real part of the slow $P$-wave number $k_{p s}$

$$
\bar{k}(\omega)=\sqrt{\frac{\eta}{2 \kappa_{0} N} \omega .}
$$

The imaginary part of the wave number yields the attenuation coefficient $\gamma$ and the reciprocal quality factor $Q^{-1}$, which for low-loss media can be written as

$$
Q^{-1}=2 \gamma / \mathfrak{R}\left\{\bar{k}_{p}\right\}=2 \mathfrak{T}\left\{\bar{k}_{p}\right\} / \mathfrak{R}\left\{\bar{k}_{p}\right\} .
$$

Then, from (1) we find

$$
Q^{-1}(\omega)=4 \Delta_{1} \bar{k}^{2} \int_{0}^{\infty} r B(r) \exp [-\bar{k} r] \cos (\bar{k} r) d r .
$$

From Eqs. (9) and (12) the meaning of the coefficients (2) and (3) becomes clear. The attenuation $Q^{-1}$ and the frequency-dependent part of $v$ are proportional to $\Delta_{1}$. Thus $\Delta_{1}$ is the measure of the magnitude of attenuation and velocity dispersion, that is, the dynamic effects. In contrast, $\Delta_{2}$ produces a frequency-independent velocity shift in (9).

In the following we analyze general properties of velocity and attenuation as given by Eqs. (9) and (12), and com- 
pute closed-form expressions for attenuation and phase velocity for several specific correlation models.

\section{GENERAL PROPERTIES OF ATTENUATION AND VELOCITY DISPERSION}

\section{A. Alternative representation using the fluctuation spectrum}

To gain further insight into the general properties of the results for attenuation and velocity dispersion (and to analyze their analytic structure, cf. the next section) it is useful to express the equations (9) and (12) in terms of the fluctuation spectrum (power spectrum), that is, the spatial Fourier transform of the correlation function. In statistically isotropic random media the fluctuation spectrum $\Phi(\kappa)$ and the correlation function $B(r)$ are related through the threedimensional Hankel transform

$$
B(r)=\frac{4 \pi}{r} \int_{0}^{\infty} \kappa \Phi(\kappa) \sin (\kappa r) d \kappa .
$$

Note that Eq. (13) implies that the integral over the fluctuation spectrum is finite if $B(0)$, i.e., the variance is finite. Substituting expression (13) into Eqs. (9) and (12), changing the order of integration, and integrating over $r$ we obtain

$$
v(\omega)=v_{0}\left[1-\Delta_{2}+16 \pi \Delta_{1} \int_{0}^{\infty} \frac{\bar{k}^{4} \kappa^{2}}{4 \bar{k}^{4}+\kappa^{4}} \Phi(\kappa) d \kappa\right]
$$

and

$$
Q^{-1}(\omega)=16 \pi \Delta_{1} \int_{0}^{\infty} \frac{\bar{k}^{2} \kappa^{4}}{4 \bar{k}^{4}+\kappa^{4}} \Phi(\kappa) d \kappa .
$$

From Eq. (15) we observe that the dynamic behavior of attenuation is controlled by the integrand, that is, by the product of fluctuation spectrum $\Phi(\kappa)$ and the function

$$
\Theta(\kappa, \bar{k})=\frac{\bar{k}^{2} \kappa^{4}}{4 \bar{k}^{4}+\kappa^{4}} .
$$

The function $\Theta(\kappa, \bar{k})$ acts like a filter and controls which part of the fluctuation spectrum yields a relevant contribution to attenuation. A similar filter function can be deduced from Eq. (14). In analogy to the acoustic scattering problem ${ }^{13}$ we refer to $\Theta$ as the spectral filter function. Analyzing the product $\Phi \Theta$ in terms of the dimensionless, spatial wave number $\kappa a$ ( $a$ is the characteristic length scale of the inhomogeneities) we identify three different regimes for different values of $\bar{k} a$.

If $\bar{k} a \ll 1$, then $\Theta(\kappa a, \bar{k} a)$ behaves like $\bar{k} a$ and, therefore, the product $\Phi \Theta$ and, hence, the attenuation, becomes small. Since $\bar{k}$ is inverse proportional to the diffusion wavelength $\lambda_{d}=\sqrt{\kappa_{0} N / \omega \eta}$, this case corresponds to the relaxed or low-frequency regime where the induced pore pressure is equilibrated during one wave cycle.

In the opposite case, if $\bar{k} a \gg 1$, then $\Theta(\kappa a, \bar{k} a)$ $\approx(\kappa a)^{4} /(\bar{k} a)^{2}$. This means that the contribution of $\Phi$ at small spatial wave numbers is suppressed but its contribution at large wave numbers is amplified. However, since $\Phi(\kappa)$ becomes very small for large $\kappa$, the product of $\Phi$ and $\Theta$

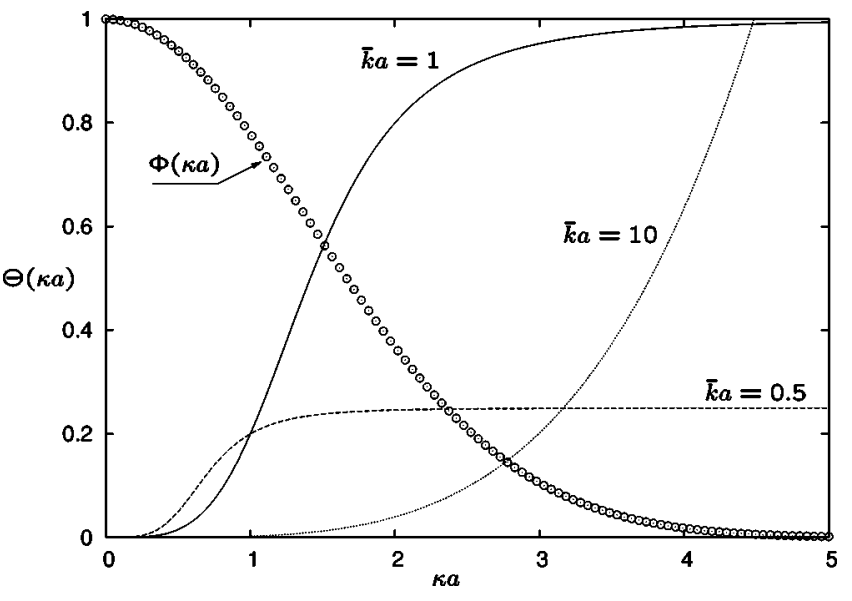

FIG. 1. The spectral filter $\theta$ as a function of dimensionless spatial wave number $\kappa a$ for varying $\bar{k} a$. The general behavior of the fluctuation spectrum $\Phi$ is also shown (circles). Elastic wave attenuation due to fluid flow is proportional to the product of $\Phi$ and $\Theta$. Maximal attenuation occurs at $\bar{k} a$ $\approx 1$.

becomes small again. In other words, in the high-frequency (unrelaxed) regime only the behavior of $\Phi$ at large $\bar{k} a$ is important.

There is an intermediate regime with $\bar{k} a \approx 1$ where $\Phi \Theta$ (and $Q^{-1}$ ) attains its maximum. Since in our approximation attenuation due to wave-induced flow and the process of conversion scattering from fast into slow $P$-waves are equivalent, maximum attenuation is observed at the "resonance" condition $\lambda_{d}=a$.

The interplay between $\Phi$ and $\Theta$ is illustrated in Fig. 1 .

\section{B. The causal relationship between attenuation and dispersion}

It can be shown that in any passive, linear medium the attenuation and phase of a wave are mutually related by the Kramers-Kronig relations ${ }^{14}$ or, more generally, satisfy a dispersion relation with $n$ subtractions (for a recent exposition of these theorems we refer to Mobley et al. ${ }^{15}$ ). Mathematically this means that the attenuation coefficient and the phase velocity, or the real and imaginary part of the complex wave number (1), are related through a Hilbert transform. Physically, this relation between attenuation and dispersion is a consequence of the causality of a pulse signal. The propagating pulse $p$ can be represented as a convolution integral

$$
p(z, t)=\int_{-\infty}^{\infty} h\left(z-z^{\prime}, t-t^{\prime}\right) s\left(z^{\prime}, t^{\prime}\right) d t^{\prime},
$$

where $h(L, \tau)$ is the impulse response and $s$ is the source wavelet. Causality implies that $h(L, \tau<0)=0$.

In our model the Fourier transform of the impulse response, $\tilde{h}(L, \omega)$, is given by $\exp \left[i \bar{k}_{p} L\right]$, where $\bar{k}_{p}$ is the complex $P$-wave number [Eq. (1)]. Instead of $\tilde{h}$, we analyze the analytic properties of its logarithmic decrement $\tilde{\gamma}(\omega)$ $\equiv \ln \tilde{h} / L=i \bar{k}_{p}$. The real and imaginary parts of $\tilde{\gamma}(\omega)$ form a Hilbert transform pair provided that $\widetilde{\gamma}(z=\omega+i y)$ is analytic in the complex upper-half plane (minimum phase condition) and is square integrable. From Eqs. (14) and (15) we can deduce that $\tilde{\gamma}(z)$ is analytic for $y>0$. However, $\tilde{\gamma}$ is not 
square integrable: Eq. (14) shows that $\tilde{\gamma}(\omega)$ diverges as $\omega^{1}$ if $\omega \rightarrow \infty$. To circumvent this difficulty we use the method of subtractions ${ }^{15}$ and form a new complex function $\Lambda$ which is square integrable:

$$
\Lambda(\omega)=\frac{\tilde{\gamma}(\omega)}{\omega^{2}}-\frac{\tilde{\gamma}(0)}{\omega^{2}}-\left.\frac{1}{\omega} \frac{d}{d \omega} \tilde{\gamma}(\omega)\right|_{\omega_{0}=0} .
$$

The function $\Lambda(\omega)$ is analytic in the upper-half complex $z$-plane. But also, $\Lambda(\omega)$ is convergent to zero as $|z| \rightarrow \infty$ and square integrable. The function $\Lambda$ forms a so-called dispersion relation with two subtractions and subtraction frequency $\omega_{0}=0 .{ }^{15}$ The real and imaginary components of $\Lambda$ form a Hilbert transform pair

$$
\begin{aligned}
& \mathfrak{R} \Lambda(\omega)=\mathcal{H}[\mathfrak{T} \Lambda(\omega)]=\frac{1}{\pi} P \int_{-\infty}^{\infty} d \omega^{\prime} \frac{\mathfrak{T} \Lambda\left(\omega^{\prime}\right)}{\omega^{\prime}-\omega}, \\
& \mathfrak{T} \Lambda(\omega)=-\mathcal{H}[\mathfrak{R} \Lambda(\omega)]=-\frac{1}{\pi} P \int_{-\infty}^{\infty} d \omega^{\prime} \frac{\mathfrak{R} \Lambda\left(\omega^{\prime}\right)}{\omega^{\prime}-\omega},
\end{aligned}
$$

where $P \int$ denotes the Cauchy principal value of the integral. Using expressions (14) and (15) the real and imaginary parts of $\Lambda$ can be written as

$$
\begin{aligned}
& \mathfrak{R} \Lambda(\omega)=-c \int_{0}^{\infty} d \kappa \Phi(\kappa) \frac{g(\kappa)}{\omega^{2}+g(\kappa)}, \\
& \mathfrak{T} \Lambda(\omega)=-c \int_{0}^{\infty} d \kappa \Phi(\kappa) \frac{\sqrt{g(\kappa)} \omega}{\omega^{2}+g(\kappa)},
\end{aligned}
$$

where $g(\kappa)=\kappa^{4} \kappa_{0}^{2} N^{2} / \eta^{2}$ and $c=4 \pi \Delta_{1} \eta /\left(v_{0} \kappa_{0} N\right)$. It is easy to verify that (21) and (22) are related through (19) and (20) if we remember the basic Hilbert transform pair $\mathcal{H}\left[-x /\left(1+x^{2}\right)\right]=-1 /\left(1+x^{2}\right)$.

Thus, the attenuation coefficient and phase velocity satisfy a twice-subtracted dispersion relation. In other words, formulas (19) and (20) allow us to compute attenuation and dispersion from each other. It is important to note that this causal relationship is an intrinsic property of our model and not a prerequisite.

\section{ANALYTICAL EXPRESSIONS AND MODELING CHOICES}

\section{A. Analytical expressions}

We now give explicit results for $Q^{-1}$ and $v$ for several correlation functions of practical interest. Ideally, the correlation function should be inferred from experimental data such as x-ray images of rock samples. In many circumstances the true correlation behavior can be well approximated by simple correlation functions such as an exponential correlation function or combinations of them. A review of frequently used correlation functions in random media is provided by Klimes. ${ }^{16}$

First, let us assume that the inhomogeneities are exponentially correlated so that $B(r)$ becomes

$$
B(r)=\exp [-|r| / a] .
$$

Here $a$ denotes the correlation length, i.e., a characteristic length scale associated with the inhomogeneities. More precisely, the correlation length $a$ is the length scale at which $B(r)$ assumes the value $e^{-1}$. The choice of a single correlation function $B(r)$ implies that the correlation length is the same for the three random functions $H(\mathbf{r}), G(\mathbf{r})$, and $C(\mathbf{r})$ (note that in general the results of paper I allow the use of different correlation lengths associated with each random function). Substituting correlation function (23) into Eqs. (9) and (12) we find

$$
Q^{-1}(\omega)=\Delta_{1} \frac{4(a \bar{k})^{2}(2 \bar{k} a+1)}{\left(1+2 \bar{k} a+2 \bar{k}^{2} a^{2}\right)^{2}}
$$

and

$$
v(\omega)=v_{0}\left[1+\Delta_{1} \frac{4(a \bar{k})^{3}(1+\bar{k} a)}{\left(1+2 \bar{k} a+2 \bar{k}^{2} a^{2}\right)^{2}}-\Delta_{2}\right] .
$$

For the so-called Gaussian correlation function

$$
B(r)=\exp \left[-r^{2} / a^{2}\right],
$$

we obtain

$$
\begin{aligned}
& Q^{-1}(\omega)= 2 \Delta_{1}(a \bar{k})^{2}\left[1-\frac{\sqrt{\pi}}{4} \sum_{z=z_{-}}^{z_{+}} a \bar{k} z\right. \\
&\left.\times \exp \left[(a \bar{k} z)^{2} / 4\right] \operatorname{erfc}[a \bar{k} z / 2]\right], \\
& v(\omega)=v_{0}\left[1+\Delta_{1}(a \bar{k})^{2} \frac{\sqrt{\pi}}{4} \sum_{z=z_{-}}^{z_{+}} a \bar{k} z^{*}\right. \\
&\left.\times \exp \left[(a \bar{k} z)^{2} / 4\right] \operatorname{erfc}[a \bar{k} z / 2]-\Delta_{2}\right],
\end{aligned}
$$

where $z_{+}=1+\mathrm{i}, z_{-}=1-\mathrm{i}, z^{*}$ denotes complex conjugation, and erfc is the complementary error function. Another widely used correlation model is the von Kármán function

$$
B(r)=2^{1-\nu} \Gamma^{-1}(\nu)\left(\frac{r}{a}\right)^{\nu} \mathrm{K}_{\nu}(r / a)
$$

where $\mathrm{K}_{\nu}$ is the modified Bessel function of third kind (MacDonald function) and $\Gamma$ denotes the gamma function. The von Kármán correlation function involves an additional parameter, the so-called Hurst coefficient $\nu$ which is assumed to be $0<\nu \leqslant 1$. For the case $\nu=\frac{1}{2}$ the von Kármán function is identical to the exponential correlation function (23). Its fluctuation spectrum is given by

$$
\Phi(\kappa)=\frac{a^{3} \Gamma\left(\nu+\frac{3}{2}\right)}{\pi^{3 / 2} \Gamma(\nu)\left(1+\kappa^{2} a^{2}\right)^{\nu+3 / 2}} .
$$

Substituting (30) into (15) and (14) gives 


$$
\begin{aligned}
Q^{-1}(\omega)= & c_{1} \Delta_{1}\left[c_{2}{ }_{3} F_{2}\left(1, \frac{1}{2}+\frac{\nu}{2}, 1+\frac{\nu}{2} ; \frac{1}{4}, \frac{3}{4} ;-4(a \bar{k})^{4}\right)\right. \\
& -\frac{1}{2} \Gamma\left(\nu+\frac{3}{2}\right)(2 \nu+3) B^{-3 / 4-\nu / 2} \cos \left[\left(\frac{3}{4}+\frac{\nu}{2}\right) A\right] \\
& +\Gamma\left(\nu+\frac{5}{2}\right) B^{-5 / 4-\nu / 2}\left\{2(a \bar{k})^{2} \cos \left[c_{3} A\right]\right. \\
& \left.\left.+\sin \left[c_{3} A\right]\right\}\right] \\
v(\omega)= & v_{0}\left[1-\Delta_{2}-\frac{c_{1}}{2} \Delta_{1}\left(-4 c_{2}(1+\nu)(a \bar{k})^{2}{ }_{3} F_{2}(1,1\right.\right. \\
+ & \left.\frac{\nu}{2}, \frac{3}{2}+\frac{\nu}{2} ; \frac{3}{4}, \frac{5}{4} ;-4(a \bar{k})^{4}\right) \\
+ & \frac{1}{2} \Gamma\left(\nu+\frac{1}{2}\right)(2 \nu+3) c_{3} B^{-3 / 4-\nu / 2} \cos \left[\left(\frac{3}{4}+\frac{\nu}{2}\right) A\right] \\
+ & \Gamma\left(\nu+\frac{3}{2}\right)\left(\frac{3}{2}+\nu\right) B^{-5 / 4-\nu / 2}\left\{2(a \bar{k})^{2} \cos \left[c_{3} A\right]\right. \\
+ & \left.\left.\left.\sin \left[c_{3} A\right]\right\}\right)\right]
\end{aligned}
$$

where we used $c_{1}=16 \sqrt{\pi}(a \bar{k})^{3} /(\Gamma(\nu)(2 \nu+3))$, $c_{2}=\Gamma(\nu+1)(2 \nu+3) /(2 \sqrt{\pi} a \bar{k}), \quad c_{3}=(1 / 4+\nu / 2), \quad A$ $=2 \arctan \left(2 a^{2} \bar{k}^{2}\right)$, and $B=1+4(a \bar{k})^{4} \cdot{ }_{3} F_{2}$ is the generalized hypergeometric function.

\section{B. Modeling choices}

Let us consider various scenarios how mesoscopic inhomogeneities can affect attenuation and dispersion of $P$ waves. The above results allow us to model signatures of wave-induced flow due to fluctuations in the solid phase, drained frame, and fluid parameters. In all numerical examples we assume that the background material is a porous sandstone with parameters specified in Table I.

In the first example, we assume that the correlation function is of exponential type (23) with varying correlation length $a$. Further, we assume that there are fluctuations of all bulk moduli and the shear modulus specified through their variances: $\sigma_{K_{d} K_{d}}^{2}=0.12, \sigma_{K_{g} K_{g}}^{2}=0.02, \sigma_{G G}^{2}=0.1$, and $\sigma_{K_{f} K_{f}}^{2}$ $=0.14$. The fluctuations of $K_{d}, K_{g}$, and $G$ are fully correlated so that the coefficient of correlation for two different random fields $R=\sigma_{X Y}^{2} / \sqrt{\sigma_{X X}^{2} \sigma_{Y Y}^{2}}$ is equal to one. In our case, the cross variances become $\sigma_{K_{d} K_{g}}^{2}=0.049, \sigma_{K_{d} G}^{2}=0.110$, and $\sigma_{G K_{g}}^{2}=0.048$. The fluctuations of porous material parameters and fluid bulk modulus are uncorrelated. Using these variances we compute the variances of the poroelastic parameters $H, C$, and $G$ : $\sigma_{H H}^{2}=0.051, \sigma_{C C}^{2}=0.081, \sigma_{H G}^{2}=0.098$, $\sigma_{H C}^{2}=0.025$, and $\sigma_{G C}^{2}=0.098$. The frequency dependence of attenuation and phase velocity for this model according to Eqs. (24) and (25) is shown in Fig. 2. The frequency is normalized by Biot's critical frequency $f_{c}$. From Fig. 2 we can observe that even weak fluctuations of the bulk moduli
TABLE I. Parameters of the background solid and fluid phases used for the computation of the numerical examples.

\begin{tabular}{cc}
\hline Porous material & \\
$K_{g}(\mathrm{GPa})$ & 40 \\
$K_{d}(\mathrm{GPa})$ & 4.5 \\
$G(\mathrm{GPa})$ & 9 \\
$\rho_{g}\left(\mathrm{~kg} / \mathrm{m}^{3}\right)$ & 2650 \\
$\phi$ & 0.17 \\
$\kappa_{0}(\mathrm{mD})$ & 250 \\
Pore fluid & \\
$K_{f}(\mathrm{GPa})$ & 2.17 \\
$\eta(\mathrm{Pa} \mathrm{s})$ & 0.001 \\
$\rho_{f}\left(\mathrm{~kg} / \mathrm{m}^{3}\right)$ & 1000 \\
& \\
Poroelastic parameters & \\
$P_{d}(\mathrm{GPa})$ & 16.5 \\
$\alpha$ & 0.89 \\
$M(\mathrm{GPa})$ & 10.4 \\
$H(\mathrm{GPa})$ & 24.7 \\
$N(\mathrm{GPa})$ & 6.9 \\
$\omega_{B}(\mathrm{kHz})$ & 680
\end{tabular}
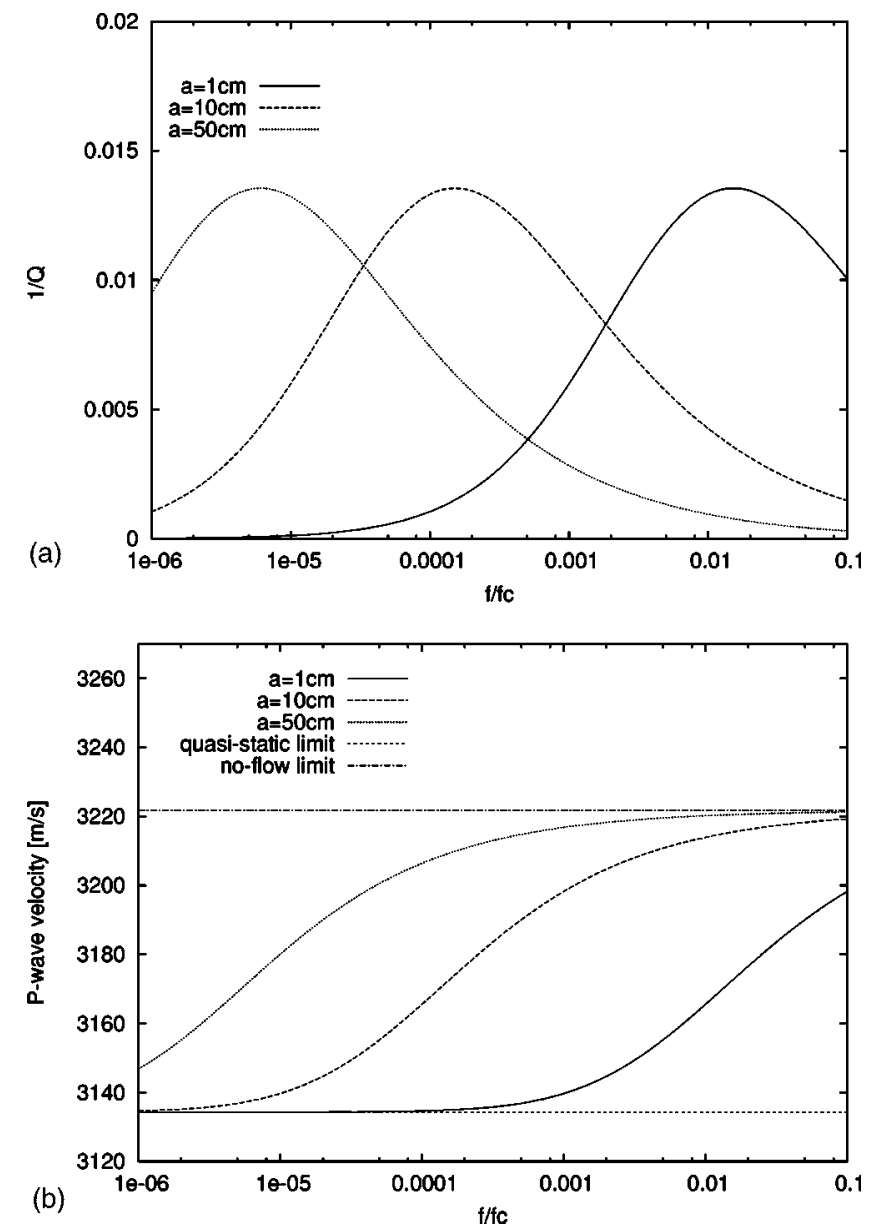

FIG. 2. (a) Reciprocal quality factor as a function of frequency (normalized by Biot's critical frequency $f_{c} \approx 100 \mathrm{kHz}$ ) for different correlation lengths. (b) $P$-wave velocity versus frequency for the same models. It can be observed that for larger correlation lengths the dispersion curves are shifted toward lower frequencies. The horizontal curves denote the quasi-static and no-flow limits, respectively. 

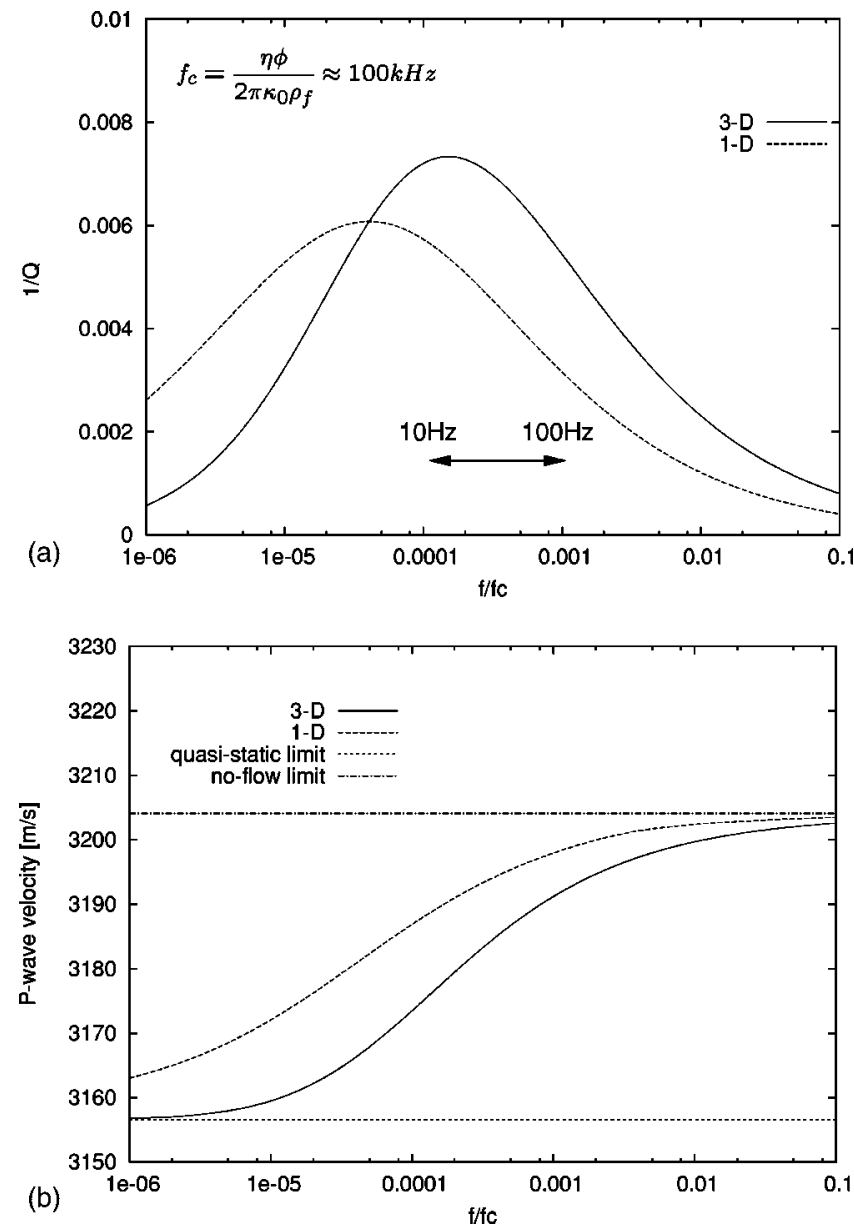

FIG. 3. Attenuation in terms of $Q^{-1}$ (a) and velocity dispersion (b) as a function of normalized frequency for a model with fluctuations in the parameter $K_{f}$ with $\sigma_{K_{f} K_{f}}^{2}=0.2$ and $a=25 \mathrm{~cm}$. For the same parameters the result of the 1-D poroelastic extension of the ODA theory is also shown (dashed curve).

can produce significant attenuation of the fast $P$ wave $\left(Q^{-1} \gtrsim 0.01\right)$.

We also compare the result for the attenuation with those obtained in the 1-D case. For this purpose we use the results of the poroelastic extension of the O'Doherty-Anstey theory [Eq. (17) in Gelinsky et al. ${ }^{7}$ ]. For the same sandstone model as above we compute $Q^{-1}$ in the case that only parameter $K_{f}$ exhibits fluctuations with $\sigma_{K_{f}}^{2}=0.2$ and $a=25 \mathrm{~cm}$ (Fig. 3). It can be observed that the magnitude of attenuation in 1-D and $3-\mathrm{D}$ random media is of the same order. However, note that the attenuation in 3-D is slightly larger. Maximal attenuation in the 3-D case is observed at

$$
\omega_{\max }^{3 D}=2 \kappa_{0} N / a^{2} \eta,
$$

whereas in the 1-D case it is observed at $\omega_{\max }^{1 D}=\kappa_{0} N / a^{2} \eta$. Thus, the maximum of attenuation in 3-D occurs at a frequency twice as large as compared with the 1-D case. In our example, this difference has important implications for the observability of the attenuation mechanism: For typical seismic frequencies $(10-100 \mathrm{~Hz})$ the attenuation due to waveinduced fluid flow is larger in 3-D inhomogeneous structures (this is indicated by the arrow in Fig. 3). It can be also observed that the low- and high-frequency velocities coin-
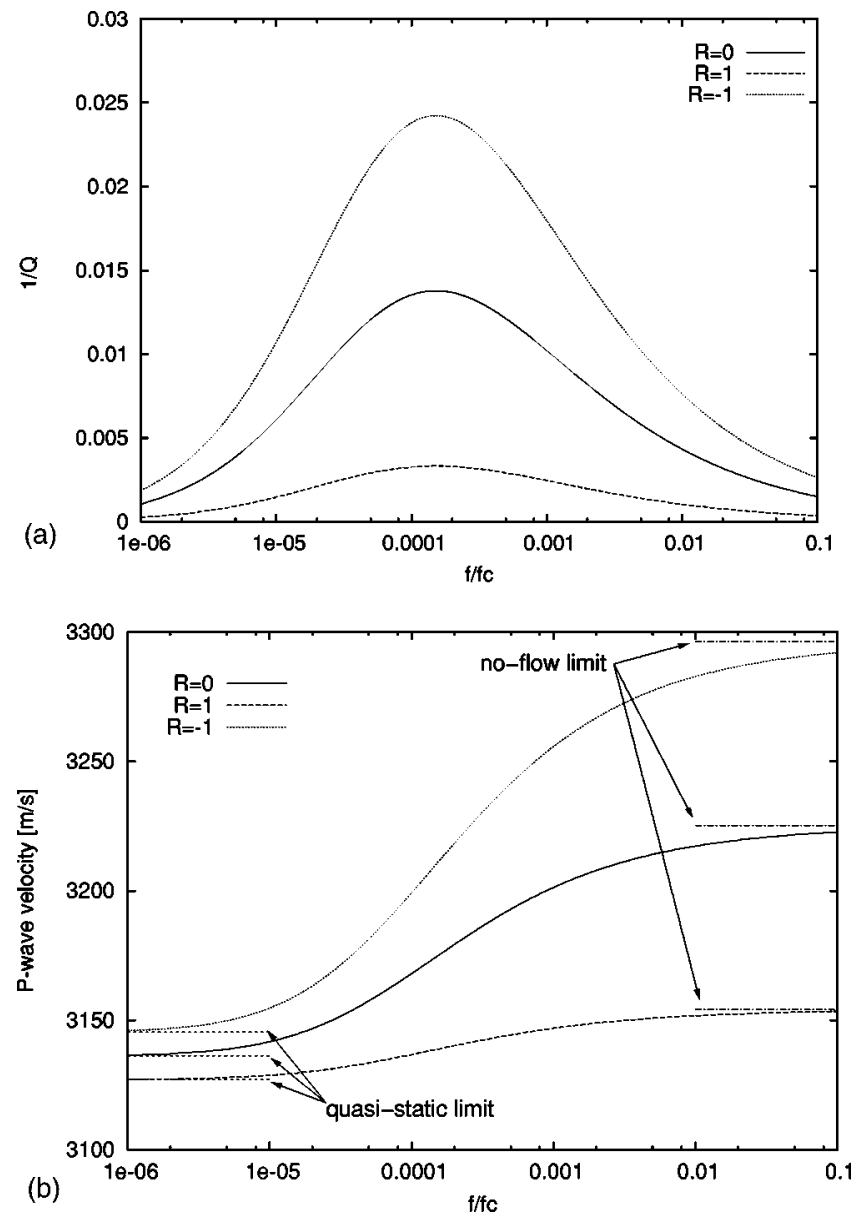

FIG. 4. (a) $Q^{-1}$ versus normalized frequency for differently correlated fluctuations. A significant amount of attenuation $\left(Q^{-1}>0.01\right)$ can be observed if the fluctuation of the solid and frame material are negatively correlated with the fluctuations of the fluid bulk modulus (that means a soft frame inhomogeneity contains a fluid with increased bulk modulus). The correlation length is constant ( $a=25 \mathrm{~cm}$ ). (b) $P$-wave velocity for the same model. It can be observed that for negative cross correlation the dispersion effect is most pronounced.

cide for the two cases. This is, however, a consequence of the constant shear modulus in this example (see also next section).

Next, we consider the influence of the cross correlations of the fluctuations. Obviously, if there is an inhomogeneity with low $P$-wave modulus but relatively high fluid bulk modulus (that is, negatively correlated fluctuations in $K_{d}$ and $K_{f}$ ), we expect an increased wave-induced fluid flow during the compression cycle of the wave. This means that both the dispersion and attenuation characteristics should be more pronounced than in the case of uncorrelated fluctuations. Such a behavior can be observed in Fig. 4, where $P$-wave velocity and attenuation are computed for the above sandstone model with $\sigma_{K_{d} K_{d}}^{2}=0.10, \sigma_{K_{g} K_{g}}^{2}=0.02, \sigma_{G G}^{2}=0.08$, and $\sigma_{K_{f} K_{f}}^{2}=0.16$. The fluctuations of $K_{d}, K_{g}$, and $G$ are positively correlated. The fluctuations of the fluid bulk modulus and those of all other fluctuating parameters are either positively (coefficient of correlation $R=1$ ) or negatively $(R=-1)$ correlated. The case of uncorrelated fluctuations $(R=0)$ between $K_{f}$ and all other moduli is also displayed in Fig. 4. We note that such scenarios may produce a 

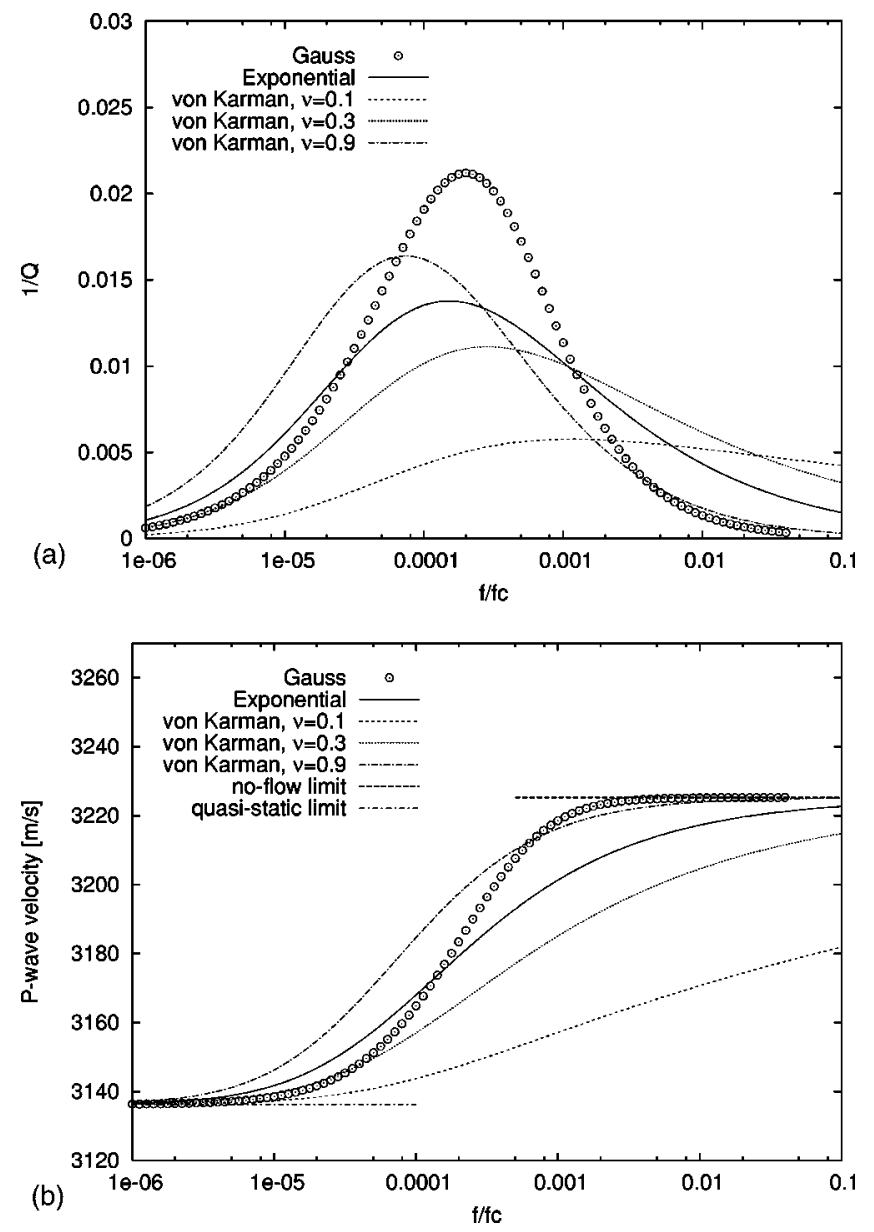

FIG. 5. $Q^{-1}$ and $P$-wave velocity as a function of normalized frequency for Gaussian, exponential, and von Kármán (the Hurst coefficient $\nu$ is denoted in the legend) correlation functions. The model parameters are the same as in Fig. 4, where the fluctuations of $K_{f}$ are uncorrelated with those of $K_{d}, G$ and $K_{g}$.

significant amount of $P$-wave attenuation $\left(0.01<Q^{-1}\right.$ $<0.1)$ even if the relative fluctuations in the medium parameters are small.

To demonstrate the influence of the correlation function on the frequency dependence of attenuation and velocity dispersion, in Fig. 5 we show $Q^{-1}$ and $P$-wave velocity for exponential, Gaussian, and von Kármán correlation functions. Note that all curves are generated using the same medium parameters (those from Fig. 4). The resulting differences in magnitude and frequency dependence of attenuation are only due to the use of a different correlation model [see Eqs. (23), (26), and (29)]. Maximal attenuation is obtained for the Gaussian correlation model. Whereas at low frequencies the frequency dependence is the same for all correlation models, one can observe that at high frequencies different asymptotes are obtained. Only the Gaussian correlation model is symmetric with respect to its maximum. The asymptotic frequency behavior of our attenuation model is analyzed below. The variability of both attenuation and velocity dispersion for different correlation models indicates the importance of the geometrical shape of mesoscopic inhomogeneities for the wave-induced flow.

\section{ASYMPTOTIC BEHAVIOR AT LOW AND HIGH FREQUENCIES}

\section{A. Attenuation}

One goal of our study is to infer the low- and highfrequency asymptotic behavior of attenuation due to waveinduced fluid flow. At low frequencies we can approximate the exponential in Eq. (1) by 1 because $B(r)$ will vanish before the exponential term changes noticeably from its value at small arguments. Obviously, an asymptote only exists if the resulting expression $\int_{0}^{\infty} d r r B(r)$ has a finite positive value. This is the case for a large class of correlation functions. Then we have $\gamma \propto \omega^{2}$ or, in terms of the quality factor,

$$
Q^{-1} \propto \omega .
$$

It is important to note that the same low-frequency behavior is reported for 1-D and 3-D periodic structures. ${ }^{5,8,10}$ The universality of this relation is discussed in Sec. VII.

At high frequencies only the behavior of $B(r)$ at small arguments is important. Assuming that the correlation function can be expanded in power series around the origin

$$
B(r / a)=1-r / a+O\left((r / a)^{2}\right),
$$

we can evaluate the integral in Eq. (1) and obtain

$$
Q^{-1} \propto \frac{1}{\sqrt{\omega}} .
$$

The same asymptote has been found in 1-D/3-D periodic and 1-D random structures. It is, however, important to note that the scaling (36) it not universal for any kind of disorder (see also Fig. 4). For example, the Gaussian correlation function (26) yields at high frequencies the proportionality $Q^{-1}$ $\propto 1 / \omega$, a much faster decrease of attenuation with frequency as compared to Eq. (36). The reason for this discrepancy is that the Gaussian correlation function behaves differently at small argument. Instead of (35) we have $B^{\text {Gauss }}(r / a)=1$ $+O\left(r^{2} / a^{2}\right)$, which means that on small scale $r \ll a$ the medium is almost homogeneous. As a consequence, at high frequencies the passing wave will create less fluid flow as compared with a medium characterized by (35) and, therefore, the decrease of $Q^{-1}$ with frequency is stronger. In other words, the smoother the medium at small spatial scales, the less wave-induced flow takes place.

\section{B. Velocity}

Having studied the low- and high-frequency asymptotes of the wave attenuation, we now analyze the asymptotic behavior of phase velocity in the cases of low- and high frequencies. In both cases the phase velocity has a finite limit. The physical situation, however, is different for these two limits: in the low-frequency limit, there is enough time during the wave cycle to equilibrate the induced pore pressure. We refer to this relaxed limit as "quasi-static" and denote the corresponding phase velocity as $v_{q s}$. In the high-frequency limit, there is no time to develop a wave-induced fluid flow. 
This situation is called the no-flow (unrelaxed) limit and we denote the phase velocity as $v_{n f}$. From Fig. (2) it can be observed that

$$
v_{n f} \geqslant v_{q s} .
$$

Physically, this relation can be explained by the additional stiffening of the porous frame in the no-flow limit.

From Eq. (9) it is straightforward to deduce $v_{q s}$. The low-frequency limit is obtained by neglecting the third term in (9). We obtain

$$
v_{q s}=v_{0}\left(1-\Delta_{2}\right) \text {. }
$$

The no-flow velocity is determined if we compute the limit $\omega \rightarrow \infty$ in Eq. (9). Since in the limit $\omega \rightarrow \infty$ only the value of $B$ at zero correlation lag yields a contribution [see Eq. (35)] we can replace $B(r)$ by $B(0)=1$. Thus the third term in (9) gives

$$
2 \Delta_{1} \bar{k}^{2} \int_{0}^{\infty} r \exp [-\bar{k} r] \sin (\bar{k} r) d r=\Delta_{1},
$$

so that

$$
v_{n f}=v_{0}\left(1+\Delta_{1}-\Delta_{2}\right) \text {. }
$$

From Eqs. (38) and (40) it follows that the relative magnitude of the dispersion effect is

$$
\frac{v_{n f}-v_{q s}}{v_{0}}=\Delta_{1}
$$

with $\Delta_{1}$ defined in Eq. (2). It is interesting to note that the limiting velocities do not depend either on the correlation function or on the transport properties of the porous material. In other words, $v_{q s}$ and $v_{n f}$ are independent of the geometry of the inhomogeneities.

An independent verification of the results for the limiting velocities can be obtained by the following consideration. First, we assume that only the fluid bulk modulus $K_{f}$ is fluctuating and all other parameters of the porous medium are constants. This situation may arise if the pore space is filled with different fluids. It is then expedient to change the parametrization from $(H, C, G)$ to $\left(P_{d}, \alpha, M, G\right)$ fluctuations because in the new parametrization only the parameter $M$ will vary. Both limiting cases must be consistent with Gassmann's equation (5) for the saturated bulk moduli $K_{\text {sat }}$ involving some effective medium parameters:

$$
K_{\text {sat }}^{*}=K_{d}^{*}+\left(\alpha^{*}\right)^{2} M^{*},
$$

where the superscript* denotes the effective value of the corresponding parameter. In the quasi-static limit, the averaging of the fluid bulk modulus should be done according to Wood's formula, which leads to the following effective $M^{*}$,

$$
M^{*}=\left\langle\frac{1}{M}\right\rangle^{-1},
$$

and hence

$$
v_{q s}=v_{0}\left(1-\frac{\alpha^{2} M}{2 H} \sigma_{M M}^{2}\right) .
$$

It is important to note that Wood's average can be applied regardless of the space dimension. Therefore, $v_{q s}$ for 3-D random media is exactly the same as the quasi-static velocity resulting from the so-called poroelastic Backus averaging. ${ }^{17}$ The coincidence of the quasi-static velocities in 1-D and 3-D space can be also observed in Fig. 3. In the no-flow limit the porous material behaves like an elastic composite. In the case of constant shear modulus the no-flow limit can be computed using the Hill average of the saturated $P$-wave moduli $H$ :

$$
H^{*}=\left\langle\frac{1}{H}\right\rangle^{-1}
$$

which in the weak fluctuation limit yields

$$
v_{n f}=v_{0}\left(1-\frac{\alpha^{4} M^{2}}{2 H^{2}} \sigma_{M M}^{2}\right)=v_{0}\left(1-\frac{1}{2} \sigma_{H H}^{2}\right) .
$$

We notice that Eq. (46) is also valid in 1-D random media. ${ }^{7}$ Again, the coincidence of both limiting velocities can be observed in Fig. 3.

The above consideration yields the same quasi-static and no-flow velocities as the 1-D approach and only requires the exact mixing laws of Wood and Hill. This could lead to the conclusion that the poroelastic Backus averaging, which provides the limiting velocities in 1-D inhomogeneous media, is also valid in 3-D inhomogeneous media. This is, however, merely a consequence due to neglecting fluctuations of the shear modulus. In general, we have to analyze the consistency of our limiting velocities with results obtained for 3-D inhomogeneous media. In the no-flow limit, we can use the results of Gold et al. ${ }^{18}$ They derived an expression for the effective $P$-wave velocity in weakly inhomogeneous elastic random media (see their equation $\mathrm{A}-28$ ):

$$
\begin{aligned}
v^{*}= & v_{0}\left(1-\frac{1}{2} \frac{\lambda^{2}}{(\lambda+2 \mu)^{2}} \sigma_{\lambda \lambda}^{2}-\frac{2}{3} \frac{\lambda \mu}{(\lambda+2 \mu)^{2}} \sigma_{\lambda \mu}^{2}\right. \\
& \left.-\frac{2}{5} \frac{\mu^{2}}{(\lambda+2 \mu)^{2}} \sigma_{\mu \mu}^{2}-\frac{4}{15} \frac{\mu}{(\lambda+2 \mu)} \sigma_{\mu \mu}^{2}\right),
\end{aligned}
$$

where $\lambda$ and $\mu$ denote the Lamé parameters and $\sigma_{x y}^{2}$ the (cross) variances of the fluctuations of the Lamé parameters. The no-flow limit can be obtained from Eq. (47) by replacing the Lamé parameters with their saturated values. To demonstrate the equivalence between this result and our expression for $v_{n f}$ we have to change from the $(\lambda, \mu)$ to $(H, G)$ parametrization. Using the simple relation between the relative fluctuations in both parametrizations $\varepsilon_{H}=\left(\lambda \varepsilon_{\lambda}+2 \mu \varepsilon_{\mu}\right) / H$ and $H=\lambda+2 \mu$, where $\lambda$ and $\mu$ are the saturated Lamé parameters, we obtain

$$
\begin{aligned}
v_{n f}= & v_{0}\left(1-\frac{1}{2} \sigma_{H H}^{2}+\frac{4}{3} \frac{G}{H} \sigma_{H G}^{2}-\frac{16}{15} \frac{G^{2}}{H^{2}} \sigma_{G G}^{2}\right. \\
& \left.-\frac{4}{15} \frac{G}{H} \sigma_{G G}^{2}\right),
\end{aligned}
$$

which is exactly Eq. (40). In conclusion, the no-flow velocity according to our approach can be obtained from the effective velocity in elastic random media when the elastic moduli are replaced by their saturated counterparts. 


\section{DISCUSSION}

\section{A. Applicability to real porous materials}

The model proposed in this paper provides expressions for frequency-dependent attenuation and dispersion in 3-D randomly inhomogeneous porous media accounting for the effect of wave-induced fluid flow. Our results are based on perturbation theory and, therefore, are restricted to weakcontrast media. However, we think that this approximate solution reveals the exact solution's essential dependence on frequency and medium parameters. In our approach the dynamic characteristics depend on the correlation properties of the medium fluctuations. Closed-form expressions for $Q^{-1}(\omega)$ and $v(\omega)$ are obtained for several correlation functions. The form of attenuation and dispersion curves are typical for a relaxation mechanism. The low-frequency behavior of attenuation is found to be $Q^{-1} \propto \omega$, whereas at high frequencies $Q^{-1} \propto \omega^{-1 / 2}$. It is interesting to note that these asymptotes coincide with those predicted by the periodicitybased approaches. ${ }^{8,10}$ Consequently, in 3-D space the observed frequency dependency of attenuation due to fluid flow has universal character independent of the type of disorder (periodic or random). This result is somewhat unexpected if we remember that in 1-D space the attenuation asymptotes are different for periodic and random structures.

Typically in the seismic frequency band attenuation is of the order ${ }^{19} 0.01<Q^{-1}<0.1$. Despite the fact that our attenuation model is restricted to weakly inhomogeneous media, it may explain attenuation values of the order 0.01 . Our model is also restricted to mesoscopic inhomogeneities (condition 1 in paper I): $\lambda \gg a \gg a_{\text {pore }}$, where $a_{\text {pore }}$ denotes any characteristic pore-scale length. In the case $\lambda \gg a$ attenuation due to scattering on weak inhomogeneities is negligible. However, if $\lambda \gtrsim a$, scattering attenuation becomes noticeable (scattering attenuation has a maximum for $\lambda=a$ ). Such a case may occur at sonic or ultrasonic frequencies. Scattering attenuation can also be modeled in the framework of the statistical smoothing method (recall that the present approach is based on this approximation method). However, since the latter approximation describes signatures of the ensemble-averaged field, its application to the evaluation of scattering attenuation in heterogeneous rocks is limited. ${ }^{20}$ Instead, a wavefield approximation valid in single realizations of the random medium $^{21}$ should be employed. An extension of the present results with proper account of scattering attenuation (i.e., to relax the restriction $\lambda \gg a$ ) is the subject of future research.

There are a few more issues that have to be addressed in future work. In order to construct a quantitative model for the case of partial saturation with pore fluids having large contrasts in their elastic properties, the present approach has to be modified in such a way that the low- and highfrequency limits are connected with the known (exact) bounds of Gassmann-Wood and Gassmann-Hill, respectively. For 1-D random media such a model has been recently developed and used in order to interpret laboratory data. ${ }^{22}$

\section{B. Analogy with other physical systems}

As discussed in more detail in paper I, attenuation and dispersion due to mesoscopic wave-induced flow can also be described as conversion scattering of the normal (fast) $P$ wave (governed by the wave equation) into Biot's slow wave, which is governed by the diffusion equation. Such a situation is not unique to poroelasticity, but occurs in a number of physical situations. Two most obvious situations of this kind are conversion scattering of elastic waves into thermal waves in inhomogeneous thermoelastic materials, and scattering of acoustic waves into viscous waves in viscous suspensions and emulsions. It is thus interesting to compare our poroelastic results with those for poroelastic solids and viscous fluids, and find out whether they yield the same patterns of frequency dependence for 1-D and 3-D periodically and randomly inhomogeneous structures.

The theory of thermoelasticity is based on a system of coupled partial differential equations which has exactly the same structure as Biot's equations of poroelasticity. ${ }^{23,24}$ Therefore, using this correspondence, results from the theory of thermoelasticity can be translated into results for poroelasticity. In thermoelasticity, dissipation of elastic waves propagating in media with varying thermal conductivity is caused by conversion into a thermal compression wave. Physically, an elastic wave causes temperature fluctuations which create a heat flux. There are numerous works on the description of the dissipation effect in randomly inhomogeneous, thermoelastic structures. For 1-D random thermoelastic media Armstrong ${ }^{25}$ showed that attenuation in the low-frequency range scales like $Q^{-1} \propto \sqrt{\omega}$, which is the very same scaling as in the 1-D random poroelastic case. In 3-D random thermoelastic media characterized by a von Kármán correlation function Shermergor and Baryshnikov ${ }^{26}$ showed that $Q^{-1}$ $\propto \omega$ at low frequencies. This asymptotic behavior is the same as found in the present work for any correlation function. Thus the observed low-frequency asymptotes of attenuation in 1-D and 3-D thermoelastic structures are the same as in corresponding poroelastic structures.

It is also interesting to compare our results with those for acoustic signatures in suspensions of solid particles in a viscous fluid. Acoustic wave attenuation in suspensions is caused by the viscous drag between the fluid and the particles during a wave cycle, i.e., by the resistance to fluid flow around the solid particle. ${ }^{27}$ Similar to the case of waveinduced flow in poroelastic structures, it is the relative motion of fluid and solid phase that controls the dissipation process. The difference is that the solid phase in suspensions is not continuous. At low frequencies one finds, ${ }^{27}$ once again, the scaling $Q^{-1} \propto \omega$. Urick ${ }^{28}$ presented a simple attenuation formula valid for all frequencies, which agrees with experimental results in suspensions. Urick's Eq. (5) yields $Q^{-1}$ $\propto \omega$ at low frequencies and $Q^{-1} \propto 1 / \sqrt{\omega}$ at high frequencies. This is the very same scaling as obtained in exponentially correlated poroelastic random media [Eq. (24)]. Interestingly, Urick's equation for attenuation also contains a term which accounts for scattering at the suspended particles resulting in the Rayleigh scattering dependence $Q^{-1} \propto \omega^{3}$. From these comparisons we can conclude that observed frequency dependencies of attenuation (and velocity) in 1-D and 3-D periodically and randomly inhomogeneous media are common for many situations where wave field energy is dissipated 
due to energy transfer from the coherent component of a propagating wave mode into a highly dissipative wave mode.

\section{CONCLUSIONS}

(1) Attenuation and dispersion of elastic waves due to waveinduced flow between 3-D mesoscopic inhomogeneities are given by simple linear integral expressions (9) and (12) involving the autocorrelation function of heterogeneities or, alternatively, involving the power spectrum of the heterogeneities [expressions (14) and (15)].

(2) Attenuation and dispersion given by these expressions satisfy a twice-subtracted dispersion relation, and thus form causal pair. Maximal attenuation occurs at the resonance condition $\lambda_{d}=a$, i.e., if the wavelength of the Biot slow wave equals the characteristic size of the inhomogeneities.

(3) For exponential, Gaussian, and von Kármán correlation functions attenuation and velocity are given by closedform expressions.

(4) For a large class of correlation functions mesoscopic flow attenuation expressed through reciprocal quality factor scales with $\omega$ at low frequencies and with $\omega^{-1 / 2}$ for high frequencies.

(5) In the presence of fluid bulk modulus fluctuations only the velocities in the low- and high-frequency limit, $v_{q s}$ and $v_{n f}$, are consistent with Gassmann's equation: $v_{q s}$ is obtained by harmonic averaging of the fluctuating modulus prior to application of Gassmann's equation. $v_{n f}$ is given by the Hill average applied to saturated moduli.

(6) Frequency dependencies of attenuation (and velocity) in 1-D and 3-D randomly poroelastic media are the same as for random thermoelastic media and suspensions of particles in a viscous fluid.

\section{ACKNOWLEDGMENTS}

This work was kindly supported by the Deutsche Forschungsgemeinschaft (Contract No. MU1725/1-1), CSIRO Petroleum, and Center of Excellence for Exploration and Production Geophysics.

${ }^{1}$ W. F. I. Murphy, J. N. Roberts, D. Yale, and K. W. Winkler, "Centimeter scale heterogeneities and microstratification in sedimentary rocks," Geophys. Res. Lett. 11, 697-700 (1984).

${ }^{2}$ T. Cadoret, G. Mavko, and B. Zinszner, "Fluid distribution effect on sonic attenuation in partially saturated limestones," Geophysics 63, 154-160 (1998).

${ }^{3}$ J. E. White, Underground Sound: Application of Seismic Waves (Elsevier, Amsterdam, 1983).

${ }^{4}$ J. E. White, N. G. Mikhaylova, and F. M. Lyakhovitsky, "Low-frequency seismic waves in fluid saturated layered rocks," Izv., Acad. Sci., USSR, Phys. Solid Earth 11(10), 654-659 (1975).
${ }^{5}$ A. N. Norris, "Low-frequency dispersion and attenuation in partially saturated rocks," J. Acoust. Soc. Am. 94, 359-370 (1993).

${ }^{6}$ B. Gurevich and S. L. Lopatnikov, "Velocity and attenuation of elastic waves in finely layered porous rocks," Geophys. J. Int. 121, 933-947 (1995).

${ }^{7}$ S. Gelinsky, S. A. Shapiro, T. M. Müller, and B. Gurevich, "Dynamic poroelasticity of thinly layered structures," Int. J. Solids Struct. 35, 47394752 (1998).

${ }^{8}$ D. L. Johnson, "Theory of frequency dependent acoustics in patchysaturated porous media,” J. Acoust. Soc. Am. 110, $682-694$ (2001).

${ }^{9}$ S. R. Pride and J. G. Berryman, "Linear dynamics of double-porosity dual-permeability materials I. Governing equations and acoustic attenuation," Phys. Rev. E 68, 036603 (2003).

${ }^{10}$ S. R. Pride, J. G. Berryman, and J. M. Harris, "Seismic attenuation due to wave-induced flow,” J. Geophys. Res. 109(B1), B01201 (2004).

${ }^{11}$ T. M. Müller and B. Gurevich, "A first-order statistical smoothing approximation for the coherent wave field in porous random media," J. Acoust. Soc. Am. 117, 1796-1805 (2005).

${ }^{12}$ F. C. Karal and J. B. Keller, "Elastic, electromagnetic and other waves in random media,” J. Math. Phys. 5, 537-547 (1964).

${ }^{13}$ A. Ishimaru, Wave Propagation and Scattering in Random Media (Academic, New York, 1978).

${ }^{14}$ K. Aki and P. G. Richards, Quantitative Seismology: Theory and Methods (Freeman, New York, 1980).

${ }^{15}$ J. Mobley, K. Kendall, M. Hughes, C. Hall, J. Marsh, G. Brandenburger, and J. Miller, "Kramers-Kronig relations applied to finite bandwidth data from suspensions of encapsulated microbubbles," J. Acoust. Soc. Am. 108, 2091-2106 (2000).

${ }^{16}$ L. Klimes, "Correlation functions of random media," Pure Appl. Geophys. 159, 1811-1831 (2002).

${ }^{17}$ S. Gelinsky and S. A. Shapiro, "Poroelastic Backus-averaging for anisotropic, layered fluid and gas saturated sediments," Geophysics 62, 18671878 (1997).

${ }^{18}$ N. Gold, S. A. Shapiro, S. Bojinski, and T. M. Müller, “An approach to upscaling for seismic waves in statistically isotropic heterogeneous elastic media," Geophysics 65, 1837-1850 (2000).

${ }^{19}$ M. Sams, J. P. Neep, M. H. Worthington, and M. S. King, "The measurement of velocity dispersion and frequency-dependent intrinsic attenuation in sedimentary rocks," Geophysics 62, 1456-1464 (1997).

${ }^{20} \mathrm{H}$. Sato, "Amplitude attenuation of impulsive waves in random media based on travel time corrected mean wave formalsim," J. Acoust. Soc. Am. 71, 559-564 (1982).

${ }^{21}$ T. M. Müller, S. A. Shapiro, and C. M. A. Sick, "Most probable ballistic waves in random media: A weak-fluctuation approximation and numerical results," Waves Random Media 12, 223-246 (2002).

${ }^{22}$ T. M. Müller and B. Gurevich, "1-D random patchy saturation model for velocity and attenuation in porous rocks," Geophysics 69, 1166-1172 (2004).

${ }^{23}$ M. A. Biot, "Thermoelasticity and irreversible thermodynamics," J. Appl. Phys. 27, 240-253 (1956).

${ }^{24}$ A. N. Norris, "On the correspondence between poroelasticity and thermoelasticity," J. Appl. Phys. 71, 1138-1141 (1992).

${ }^{25}$ B. H. Armstrong, "Models for thermoelastic attenuation of waves in heterogeneous solids," Geophysics 49, 1032-1040 (1984).

${ }^{26}$ T. D. Shermergor and V. G. Baryshnikov, "Description of intercrystalline thermoelastic internal friction using the von Karman correlation function," Sov. Phys. Solid State 10, 1709-1711 (1969).

${ }^{27}$ R. L. Gibson and M. N. Toksöz, "Viscous attenuation of acoustic waves in suspensions," J. Acoust. Soc. Am. 85, 1925-1934 (1989).

${ }^{28}$ R. J. Urick, "The absorption of sound in suspensions of irregular particles," J. Acoust. Soc. Am. 20, 283-289 (1948). 